contextos I contribuciones e informaciones especiales 


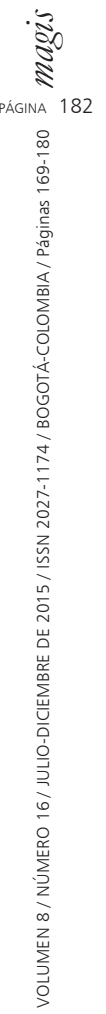




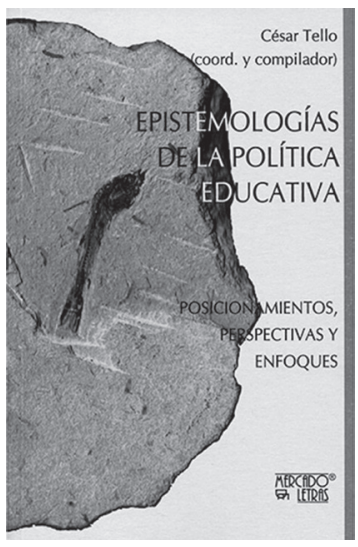

Reseña del libro

\section{Epistemologías de la política educativa: desafíos analíticos y debates teóricos en un campo emergente}

Book Review Epistemologies of Educational Policy: Analytical Challenges and Theoretical Debates in an Emerging Field

Compte-rendu du livre Épistémologies de la politique

éducative: défis analytiques et débats théoriques dans

un domaine émergeant

Resenha do livro Epistemologias da política educativa: desafios analíticos e debates teóricos em um campo emergente

Fecha de recepción: 4 DE NOVIEMBRE DE 2013 / Fecha de aceptación: 6 DE MAYO DE 2015 / Fecha de disponibilidad en línea: 30 DE OCTUBRE DE 2015

Encuentre este artículo en http://magisinvestigacioneducacionjaverianaeduco/

Escrita por Gimena Nieto Universidad Nacional de San Martín San Martín, Provincia de Buenos Aires, Argentina gimenanieto@yahoocomar

\section{Ficha bibliográfica}

Título: Epistemologías de la política educativa: posicionamientos, perspectivas y enfoques

Coordinador y compilador: César Tello Editorial: Mercado de Letras

Año: 2013

Número de páginas: 614 


\section{Contenido}

Presentación, César Tello. Primera parte, Perspectivas epistemológicas y enfoques epistemetodológicos para la investigación en política educativa. Capítulo 1. Las epistemologías de la política educativa. Notas históricas y epistemológicas sobre el campo, César Tello. Capítulo 2. El análisis crítico del discurso (ACD). Aspectos teóricos y metodológicos en la investigación de políticas educativas, Mónica Eva Pini. Capítulo 3. La interpelación de lo cotidiano a las políticas educativas (políticas educativas, reformas y vida escolar cotidiana), Gabriel Asprella. Capítulo 4. El enfoque jurídico normativo de la política educacional, Guillermo Ruiz. Capítulo 5. Las políticas educativas como textos y como discursos. El enfoque de Stephen Ball, Silvia Novick de Senén González e Isabel Vilella Paz. Capítulo 6. Estudios del discurso pedagógico y las recontextualizaciones: la perspectiva bernsteiniana para la investigación sobre políticas educativas, Oscar L. Graizer. Capítulo 7. Torres profesor, Torres consejero, Torres pedagogo: una reflexión sobre los componentes epistemológicos de la sociología política de la educación de Carlos Alberto Torres, Lauren lla Misiaszek. Capítulo 8. Un abordaje de la cuestión epistemológica en los estudios comparados sobre política educativa, Jorge M. Gorostiaga. Capítulo 9. La perspectiva epistemológica marxista de Gramsci y la investigación de políticas educacionales. Su vinculación con el rol de la universidad, Maria de Lourdes Pinto de Almeida y Sidney Reinaldo da Silva. Capítulo 10. Políticas educativas y relaciones de sexo-género, Graciela Morgade. Capítulo 11. Las contribuciones de la perspectiva marxista para la investigación sobre políticas educativas, Gisele Masson y Jefferson Mainardes. Capítulo 12. Análisis político del discurso: posiciones y significaciones para la política educativa, Myriam Southwell. Capítulo 13. La categoría de capital cultural en Pierre Bourdieu para el análisis de políticas y las prácticas educativas, Carina V. Kaplan y Pablo di Napoli. Capítulo 14. Etnografía en el análisis de políticas educativas: reflexiones epistemológicas desde América Latina, Carlos Miñana Blasco. Capítulo 15. La cartografía social y el pluralismo como enfoque epistemológico para el análisis de políticas educativas, César Tello y Jorge M. Gorostiaga. Segunda parte. Reflexiones epistemológicas y epistemetodológicas para la investigación en política educativa - Un acercamiento a la epistemología de las políticas educativas: conceptos, unidad de análisis y teoría, Pedro Flores Crespo - Políticas educativas o la transdisciplinariedad de un campo de estudio, António Teodoro - Epistemología de las políticas educativas: algunas precisiones conceptuales, Dermeval Saviani • La epistemología del oprimido y las políticas educativas, José Eustáquio Romão • La indisciplina de los campos: una mirada desde la epistemología de la política educativa, Enrique del Percio y Mercedes Palumbo - Las epistemologías de la política educativa y sus contribuciones para el campo, Jefferson Mainardes • Investigando las políticas educativas: ¿cómo teorizamos la "causalidad" entre lo macro y lo micro?, Gary Anderson y Janelle Scott • Epistemologías de la investigación y la docencia: un acercamiento desde Freire y la teoría crítica, Carlos Alberto Torres • Principales corrientes epistemológicas de educación comparada, Erwin H. Epstein - Marco metaanalítico para el análisis de las políticas educativas, Sally Power • ¿Hay algún obstáculo epistemológico para incorporar la cultura visual en la investigación educacional?, Gustavo E Fishman. Posfacio. Enfoque para el análisis de investigaciones en política educacional, Maria de Lourdes Pinto de Almeida 
El presente libro tiene la intención de poner en debate las epistemologías propias del campo de la investigación en política educativa. Mediante la metodología basada en la sistematización de investigaciones en temáticas sobre política educativa y desde una perspectiva crítica, plantea las preocupaciones y supuestos epistemológicos que subyacen en ese campo teórico.

Desde una perspectiva reflexiva, esta valiosa propuesta de debate epistemológico plantea la actividad compleja de comprender cuáles y cómo son los procesos de investigación en política educativa, y añade la necesidad de definir una serie de acuerdos con base en la categoría de "epistemología de la política educativa" para nombrar aquello que acontece en los modos de pensar y reflexionar sobre la producción del conocimiento científico.

En la presentación, el autor y compilador César Tello da cuenta de la inexistencia de perspectivas teóricas y metodológicas sobre los estudios epistemológicos de la política educativa tanto en América Latina como en Europa y Estados Unidos. Esto produce una debilidad en los procesos de investigación y una carencia en los debates, debido a la relativización de las investigaciones a marcos, fundamentaciones y referentes teóricos cuyos autores no explicitan a menudo su posicionamiento epistemológico, ético e ideológico.

Desde este metaanálisis, se formula un nuevo esquema teórico reflexivo anclado en el concepto de "epistemologías de la política educativa", el cual en primera instancia alude a los procesos de producción del conocimiento que se realizan de un modo metódico y sistemático. Este concepto refiere a un análisis de carácter científico concerniente a la investigación en epistemología de la política educativa; y lo considera como un campo teórico emergente y en construcción, impulsado por la Red Latinoamericana de Estudios Epistemológicos en Política Educativa (ReLePe)1.

\section{Organización y estructura del libro}

El libro está constituido por cinco ejes estructurales para comprender la realidad de la política educativa, a partir de varios autores con diversas perspectivas y enfoques:

a) Problemáticas y limitaciones en el desarrollo de investigación en política educativa, b) Debates epistemológicos en política educativa: perspectivas y posicionamientos, c) Epistemologías para el análisis y la investigación en política educativa, d) Historia del campo de la política educativa, e) Investigación analítica de autores referentes en política educativa.

Presenta diversas investigaciones en política educativa que autores de varios países realizaron en los últimos años, con la particularidad de generar un debate epistemológico en este campo desde sus denominaciones, entrecruzamientos teóricos y perspectivas.

A su vez, este libro se organiza en dos bloques. En el primero de carácter narrativo, mediante el despliegue de quince investigaciones, se desarrolla un recorrido histórico acerca del campo de la política educativa desde su surgimiento hasta su institucionalización, sumado a la descripción y análisis de los autores acerca de sus propios posicionamientos, perspectivas epistemológicas y enfoques epistemetodológicos para la investigación en ese campo del conocimiento.

1 Red de investigadores preocupados por la perspectiva epistemológica para el análisis de políticas educativas en sus diversas acepciones: producción de conocimiento, investigación, historia del campo, entre otros ejes de investigación. Disponible en: http://www.relepe.org

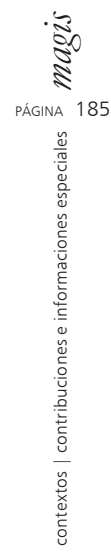

Descripción del artículo | Article description | Description de l'article | Artigo descrição

Este libro presenta las epistemologías propias del campo de la investigación en política educativa, un área específica sobre la que hay poco escrito. Como se concluye en la reseña, "el libro puede ser especialmente recomendable para teóricos interesados en estudiar, conocer y comprender mediante un punto de vista crítico cuáles son los modos en que se investiga en las Ciencias Sociales específicamente en el campo de las Epistemologías de la Política Educativa". Asimismo, por sus características puede brindar aportes significativos, actualizados y válidos a los académicos latinoamericanos implicados en un debate reflexivo acerca de la propia práctica de investigación, como así también la deconstrucción de las perspectivas actuales para la apertura de nuevos modos de producción de conocimiento. César Tello (coord.) (2013). Epistemologías de la política educativa: posicionamientos, perspectivas y enfoques. Campinas: Mercado de Letras. 
En el segundo bloque, desde un abordaje reflexivo, se aprecia el desarrollo de varias investigaciones sociales, políticas y educativas ligadas a la categoría teórica de epistemología de la política educativa. Asimismo se pueden identificar aquellos trabajos centrados en la preocupación por la configuración de un campo teórico referido a la política educativa y otros relacionados con el desarrollo de debates epistemetodológicos vinculados a contextos históricos. El cierre del libro presenta un posfacio, en el cual Maria de Lourdes Pinto de Almeida desarrolla una serie de ideas acerca de la ciencia, el conocimiento y el contexto histórico para enmarcar y contribuir al análisis de las epistemologías de la política educativa.

\section{A modo de conclusión}

Desde un punto de vista crítico, el libro invita a conocer y comprender cuáles son los modos en que se investiga en las ciencias sociales, específicamente en el campo de las epistemologías de la política educativa. Nos presenta una construcción colectiva atravesada por un pluralismo epistemológico, a partir de la formulación de ejes de análisis y nuevas categorías teóricas y analíticas que definen enfoques, posicionamientos y perspectivas para que los investigadores, docentes, estudiantes de grado y posgrado inicien un debate reflexivo acerca de la propia práctica de investigación, y también sobre la deconstrucción de las perspectivas actuales para la apertura de nuevos modos de producción de conocimiento. En síntesis, el autor propone mediante una compilación de referentes teóricos y reflexiones, el fortalecimiento y la profundización de variadas formas de trabajo investigativo dentro de este campo emergente que promete el descubrimiento de nuevas potencialidades para un desarrollo teórico y analítico acerca de las epistemologías de la política educativa.

\section{Sobre la autora}

Gimena Nieto es licenciada en educación y profesora en ciencias de la educación, Universidad Nacional de San Martín. Se desempeña como docente e investigadora de esa casa de Altos Estudios. Actualmente, está cursando estudios de doctorado en educación en las Universidades Nacionales de San Martín, Tres de Febrero y Lanús. 